\title{
Weight-based search to find clusters around medians in subspaces
}

\author{
Sergio Peignier ${ }^{1} \quad$ Christophe Rigotti ${ }^{1} \quad$ Anthony Rossi ${ }^{1}$ \\ Guillaume Beslon ${ }^{1}$ \\ ${ }^{1}$ Univ Lyon, INSA-Lyon, CNRS, INRIA, LIRIS, UMR5205, \\ F-69621, Villeurbanne, France \\ name.lastname@inria.fr
}

\begin{abstract}
There exist several clustering paradigms, leading to different techniques that are complementary in the analyst toolbox, each having its own merits and interests. Among these techniques, the K-medians approach is recognized as being robust to noise and outliers, and is an important optimization task with many different applications (e.g., facility location). In the context of subspace clustering, several paradigms have been investigated (e.g., centroid-based, cell-based), while the median-based approach has received less attention. Moreover, using standard subspace clustering outputs (e.g., centroids, medoids) there is no straightforward procedure to compute the cluster membership that optimizes the dispersion around medians. This paper advocates for the use of median-based subspace clustering as a complementary tool. Indeed, it shows that such an approach exhibits satisfactory quality clusters when compared to well-established paradigms, while medians have still their own interests depending on the user application (robustness to noise/outliers and location optimality). This paper shows that a weight-based hill climbing algorithm using a stochastic local exploration step can be sufficient to produce the clusters.
\end{abstract}

\section{Introduction}

Clustering is a data mining task that aims to group objects sharing similar characteristics over the whole data space. There exist various clustering algorithms relying on different similarity measures and paradigms. They provide complementary tools to the analyst, each having its own merits and interests. Among the major categories, clustering-oriented approaches [13] group objects mainly using distance-based similarities and tend to build center-based hyperspherical shaped clusters. For example the very well known K-means algorithm relies on the Euclidean distance and produces clusters around centroids. Other techniques known as the K-medians approaches [4], use the Manhattan distance to group data objects around medians that are less sensitive to unusual 
and extreme values and more robust to noise and outliers [12]. Moreover according to [1] the use of the Manhattan distance should be preferred for high dimensional data mining applications, since this metric is less impacted by the problem known as the curse of dimensionality. Beside these interesting properties, the K-medians problem is an important optimization task with many different applications.

Given a set of objects in a $D$-dimensional space, K-medians clustering aims to partition the given objects into K clusters so as to minimize the sum of the Manhattan distances between each object and the median of its cluster [4]. This technique has been applied for instance to facility locations (e.g., finding optimal location for resource storage). K-medians have also been used to clusterize histograms and then retrieve consensus histograms [9]. More recently K-medians have also been used for data allocation in communication networks [15].

Various concepts have been investigated in the subspace clustering community. However despite their importance, medians seem to have received less attention than other clustering paradigms. Literature reviews (e.g., $[8,14])$ do not report subspace clustering algorithms based on medians and such an approach has not been investigated in more recent works either (e.g., $[18,10])$. In addition, even if various subspace clustering techniques have been developed to build groups of objects around centroids or medoids, there is no straitforward procedure to use these groups to compute cluster memberships that optimize the dispersion around medians.

The main contribution of this paper is to show that a pure median-based subspace clustering exhibits satisfactory clusters when compared to well-established paradigms (using the evaluation framework of [13]). This advocates for its use as a complementary tool in the family of the subspace clustering approaches, since medians have their own interests depending on the user application, notably their robustness to noise/outliers and their inherent location optimality (e.g., for facility location).

This paper shows that even an easy to implement strategy is promising to find such subspace clusters. Indeed a simple hill climbing algorithm based on stochastic local exploration steps can be used to produce the clusters. As many algorithms that aim at producing hyper spherical clusters (or subspace clusters) by minimizing dispersion (e.g., K-means, PROCLUS [2]), the algorithm presented here, can be stuck in local minima and different clusterings can be obtained for different executions (due to some stochastic steps). So, as for the other algorithms, the one presented in this paper needs to be launched several times.

The presented algorithm relies on three key hints: 1) New guesses for candidate median locations can be obtained directly from the data objects themselves. 2) The algorithm uses a weight-based strategy to guide its local search towards promising subspace clusters. 3) In order the guide the search and build the candidate median locations, it turns out that using a dynamic sample of the dataset is sufficient. This allows thus to reduce the amount of data to handle at each iteration, while the appropriateness of sampling based strategies remains an open question for many subspace clustering approaches. 


\section{Related works}

\subsection{Median-based clustering}

The K-medians problem (e.g., [4]) is a well defined and NP-hard problem that is a research topic of interest for both the computational geometry and the clustering communities. This problem has been studied in the computational geometry domain with the aim to find optimal locations for centers and facilities in order to minimize costs. We refer the reader to [6] for examples of real world applications of K-medians. On the other hand the clustering community studied the K-medians problem in order to develop techniques to find clusters that are robust to noise and outliers. The K-medians clustering and facility location tasks have been investigated from the perspective of combinatorial optimization, approximation algorithms, worst-case and probabilistic analysis (e.g., [5]). However, locating centers having optimal locations or partitioning the objects of the dataset to form center-based clusters are two different ways to see the same problem.

\subsection{Subspace clustering}

The purpose of subspace clustering is not only to partition a dataset into groups of similar objects, this technique also aims to detect the subspaces where similarities occur. Therefore each cluster is associated to a particular subspace. Many approaches have been investigated for subspace clustering in the literature using various clustering paradigms. The reader is referred for instance to $[7,13,14]$ for detailed reviews and comparisons of the methods, including the main categories: Cell-based, density based, clustering-oriented approaches and also pattern-based clustering or biclustering approaches (e.g., [17]).

A subspace clustering technique based on medians belongs to the family of clustering-oriented approaches. These approaches are based on parameters specifying properties of the targeted clustering such as the expected number of clusters or the cluster average dimensionality. According to these constraints, the objects are grouped together mainly using distance-based similarities. Most of these methods tend to build hyper-spherical shaped clusters around centers (e.g., centroids, medoids). No subspace clustering technique based on medians, as the one presented in this paper, has been reported in relevant reviews, e.g., [7, 13] nor in recent subspace clustering proposals, e.g., [18, 10].

\section{Problem statement}

This section recalls some preliminary definitions and specifies the task considered in the paper. 


\subsection{Dataset and preprocessing}

Let a set of objects $\mathcal{S}=\left\{s_{1}, s_{2} \ldots\right\}$ denote a dataset. Each object in $\mathcal{S}$ has a unique identifier and is described in $\mathbb{R}^{D}$ by $D$ features (the coordinates of the object). Let $D$ denote the number of dimensions (i.e., the dimensionality) of $\mathcal{S}$. Each dimension is represented by a number from 1 to $D$ and the set of all dimensions of the dataset is denoted $\mathcal{D}=\{1, \ldots, D\}$.

To avoid being impacted by the original offsets and scales of the features, we suppose that the data have been standardized, as in many clustering frameworks. We rely here on the usual z-score standardization, leading to a mean of zero and a standard deviation equal to one for each feature.

\section{$3.2 \quad$ K-medians problem}

Given a dataset $\mathcal{S}$ in a space $\mathcal{D}$, let $\mathcal{H}=\left\{m_{1}, \ldots, m_{k}\right\}$ be a non-empty set of centers where each center $m \in \mathcal{H}$ is an element in $\mathbb{R}^{D}$. The Manhattan $\left(L_{1}\right)$ distance between two objects $u$ and $v$ in space $\mathcal{D}$ is defined as $\|u, v\|_{1}=$ $\sum_{d \in \mathcal{D}}\left|u_{d}-v_{d}\right|$. The Manhattan distance between an object $s$ and its closest center defines the so-called Absolute Error associated to $s$ for $\mathcal{H}: A E(s, \mathcal{H})=$ $\min _{m \in \mathcal{H}}\left(\|s, m\|_{1}\right)$. The K-medians problem can be formulated as the optimization problem that aims to find a set $\mathcal{H}$ of $K$ centers that minimizes the Sum of Absolute Errors of the objects in $\mathcal{S}$ defined as $S A E(\mathcal{S}, \mathcal{H})=\sum_{s \in \mathcal{S}} A E(s, \mathcal{H})$. Such a set $\mathcal{H}$ defines a partition of $\mathcal{S}$ into $K$ clusters $\mathcal{C}=\left\{C_{1}, \ldots, C_{K}\right\}$, where a cluster $C_{i}$ contains the objects of $\mathcal{S}$ for which $m_{i} \in \mathcal{H}$ is the closest center (using the Manhattan distance).

\subsection{Subspace clustering based on medians}

A subspace clustering $\mathcal{M}$, called hereafter a model, is a set of centers where each center $m_{i} \in \mathcal{M}$ is associated to a subspace $\mathcal{D}_{i}$ of $\mathcal{D}$. From the point of view of center-based subspace clustering, a cluster center described in a given subspace can be perceived informally as a summary of the cluster objects. Indeed this set of objects can be represented in a more abstract way simply by the location of the center along the dimensions considered in the cluster subspace. For a dimension $d$ that is not in $\mathcal{D}_{i}$, the intended meaning is that, along $d$, the objects of the cluster follow the same distribution as the other objects of the dataset. For an object $s$, the Absolute Error is then $A E(s, \mathcal{M})=\min _{m \in \mathcal{M}} \operatorname{dist}(s, m)$, where $\operatorname{dist}\left(s, m_{i}\right)=\sum_{d \in \mathcal{D}_{i}}\left|s_{d}-m_{i, d}\right|+\sum_{d \in \mathcal{D} \backslash \mathcal{D}_{i}}\left|s_{d}-\mu_{d}\right|$, with $m_{i, d}$ the coordinate of $m_{i}$ in dimension $d$, and with $\mu_{d}$ the mean of the location of all objects in $\mathcal{S}$ along $d$. Notice that, since the dataset is supposed to be normalized using a z-score, then $\mu_{d}=0$ for all $d$.

The $S A E$ is still defined as $S A E(\mathcal{S}, \mathcal{M})=\sum_{s \in \mathcal{S}} A E(s, \mathcal{M})$. Each object is associated to the cluster $C_{i}$ such that $\operatorname{dist}\left(s, m_{i}\right)$ is minimized. If several clusters minimize this expression then the object is non-deterministically associated to

one of them. Finally, the size of a model $\mathcal{M}$, noted $\operatorname{Size}(\mathcal{M})$, is defined as the sum of the dimensionalities of all subspaces associated to the centers in 
$\mathcal{M}$, and is interpreted as the level of detail captured by the clustering. To perform such a median-based subspace clustering, the core task considered in this paper is then to find a set of centers $\mathcal{M}$ that minimizes the $S A E$ and such that $\operatorname{Size}(\mathcal{M}) \leq S D_{\max }$, where $S D_{\max }$ is a parameter denoting the maximum Sum of Dimensions used in $\mathcal{M}$ to define all the subspaces.

\section{Algorithm}

This section introduces the SubCMedians algorithm to handle the mediansbased subspace clustering task.

\subsection{General hill climbing procedure}

Let $\mathcal{S}$ be a dataset and $\mathcal{M}$ a model (a set of centers each being defined in its own subspace). The algorithm presented in this paper is a hill climbing oriented technique that aims to minimize the objective function $S A E(\mathcal{S}, \mathcal{M})$. It updates iteratively a model using a stochastic optimization approach, while keeping the maximum model size constraint satisfied.

The hill climbing process can be perceived as a local search process on a graph of models. Each vertex $\mathcal{M}$ in the graph is a model such that $\operatorname{Size}(\mathcal{M}) \leq$ $S D_{\text {max }}$ and there is an edge from $\mathcal{M}$ to a vertex $\mathcal{M}^{\prime}$ if $\mathcal{M}^{\prime} \in \mathcal{N}$ eighbors $(\mathcal{M})$, where $\mathcal{N}$ eighbors $(\mathcal{M})$ is a function that defines the set of neighbors of $\mathcal{M}$.

The algorithm takes as initial vertex the empty model (a model containing no center), denoted $\mathcal{M}_{\emptyset}$, and for which the definition of $A E$ is extended as follows: $A E\left(s, \mathcal{M}_{\emptyset}\right)=\sum_{d \in \mathcal{D}}\left|s_{d}-\mu_{d}\right|$, all $\mu_{d}$ being still equal to 0 due to the dataset standardization.

At each iteration the algorithm evaluates the $S A E$ for a random neighbor of the current vertex, whenever the $S A E$ associated to the new node is smaller or equal to the current $S A E$, the algorithm moves towards the neighbor. The algorithm uses the data objects themselves to generate the neighborhood of a model $\mathcal{M}$. Indeed, a neighbor of a model $\mathcal{M}$ is a model that can be obtained from $\mathcal{M}$ by inserting/removing a dimension in a subspace, and setting a center coordinate to a value equal to a coordinate of an existing object. The neighbors generation is fully described in Section 5.2.

\subsection{Sampling the dataset}

An advantage of a median based subspace clustering is that the location of the median can be estimated using a sample. This is the case for other approaches (for example centroid based ones), but remains an open question for subspace clustering techniques in general. Let $X$ be a continuous random variable with a density function $f(x)$ and a median $\theta$. The median of a sample of $n$ independent realizations of $X$ is an estimator (noted $\hat{\theta}$ ) of the median of $X$ and is normally

distributed around $\theta$ with a standard deviation $\sigma_{\hat{\theta}}=\frac{1}{2 f(\theta) \sqrt{n}}[11,16]$. This approximation derives from the central limit theorem and holds for large enough 


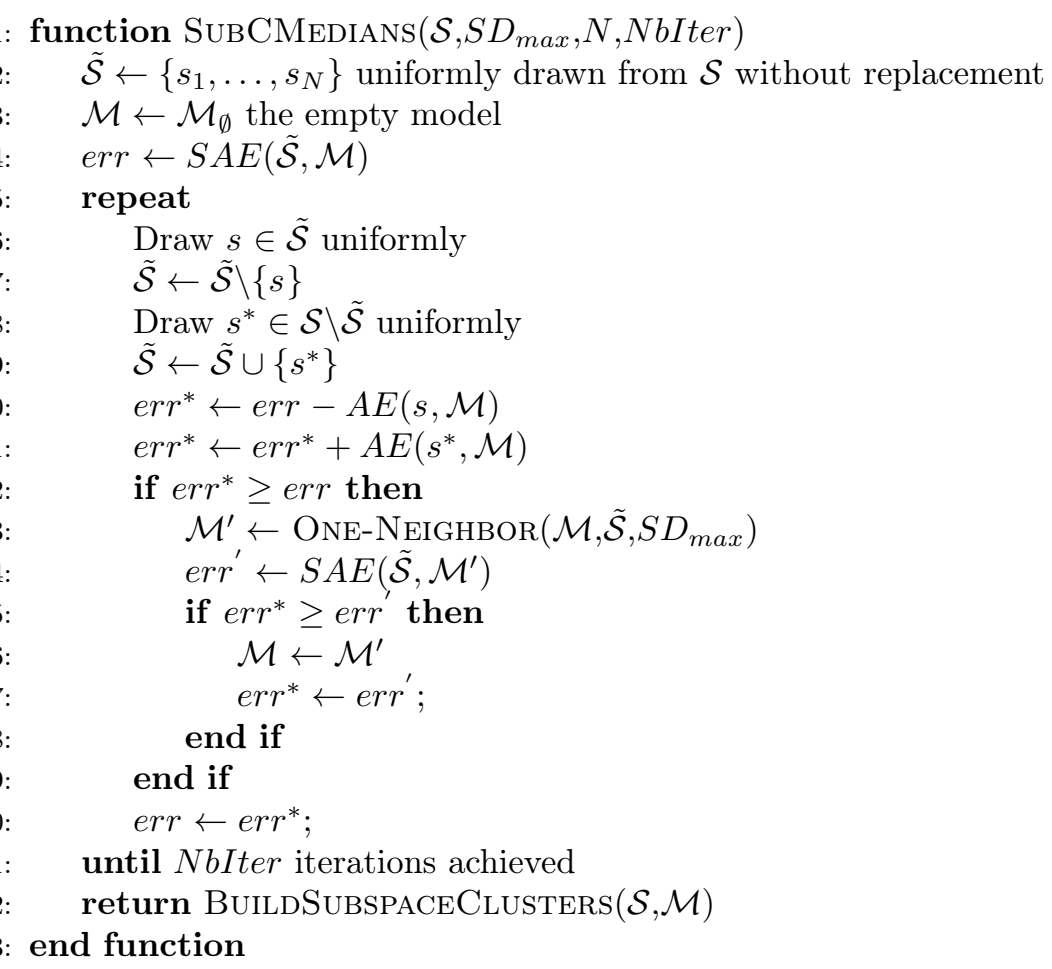

Figure 1: SubCMedians algorithm.

samples. Let us suppose that $X$ follows a Gaussian distribution $X \sim \mathcal{N}(\mu, \sigma)$, where $\mu$ and $\sigma$ denote respectively the mean and standard deviation of $X$. The density function of the distribution is $f(x)=\frac{1}{\sigma \sqrt{2 \pi}} e^{-\frac{1}{2}\left(\frac{x-\mu}{\sigma}\right)^{2}}$. Since the mean and the median of a Gaussian distribution are equal, $f(\theta)$ simplifies to $f(\theta)=\frac{1}{\sigma \sqrt{2 \pi}}$, and we have $\sigma_{\hat{\theta}}=\sigma \sqrt{\frac{\pi}{2 n}}$. So $\hat{\theta} \sim \mathcal{N}\left(\mu, \sigma \sqrt{\frac{\pi}{2 n}}\right)$, and thus a subspace clustering algorithm based on medians does not require to use the entire dataset. Indeed, a dataset sample $\tilde{\mathcal{S}} \subseteq \mathcal{S}$, should allow the algorithm to build a model without important degradation of the clustering quality, while reducing the amount of data to handle. This choice is retained in SubCMedians, but to limit the negative effects of a possible bad sample choice, the algorithm will modify dynamically the sample along the iterations.

\subsection{Lazy hill climbing procedure}

The algorithm is given in Figure 1. It takes as input a dataset $\mathcal{S}$ described in a space $\mathcal{D}$ and three parameters: the maximum model size $S D_{\max }$, the sample size $N$ and the number of iterations NbIter. The setting of these parameters is presented in Section 6.3 (technical details provided in Appendix A.3). The first step of the algorithm is to initialize the model $\mathcal{M}$, the sample $\tilde{\mathcal{S}}(N$ objects 
randomly chosen in $\mathcal{S}$ ) and to compute the error err corresponding to $\mathcal{M}$.

At each iteration, a data object picked at random in $\tilde{\mathcal{S}}$ is replaced by an object uniformly drawn from $\mathcal{S} \backslash \tilde{\mathcal{S}}$ in order to change dynamically the sample. Then (lines 10 and 11) the $S A E$ err ${ }^{*}$ on the new sample is computed incrementally by substracting the $A E$ associated to the object that has been removed and adding the $A E$ for the new object.

Next, the algorithm performs a lazy hill climbing step. Indeed, if the $S A E$ on the new sample is better (i.e., lesser) than the $S A E$ on the previous sample the algorithm does not try to improve the model itself during this iteration. Otherwise a new model $\mathcal{M}^{\prime}$ is computed using the function $\operatorname{OnE}-\operatorname{NeIGHBOR}()$ (detailed in the next section) and $\mathcal{M}^{\prime}$ is retained if it improves the $S A E$.

Finally, the algorithm outputs a subspace clustering in the form of a set of disjoint clusters and their corresponding subspaces using a call to function BuildSubspaceClusters(). This function is very simple and not detailed further, it simply associates each data object to its closest center (the one minimizing $A E$ ). If several centers give the same minimal $A E$, then one is chosen in a nondeterministic way. At the end, if a center has no associated object then it does not lead to a cluster and BuildSubspaceClusters() discards it.

\section{Neighborhood exploration heuristic}

The local exploration can be guided by the expected gain in $S A E$ associated to the optimization of the location of a given center. Intuitively adjusting the center of a cluster containing many objects and having high dispersion is likely to have a higher effect on the the $S A E$ (see Appendix A.1 for technical details).

\subsection{Weighted candidate model}

Since the clusters and their standard deviations are not known beforehand, SubCMedians uses two heuristics to favor some of the centers that could be most promising. The first one is to guide the local exploration by modifying the current model with a coordinate of a uniformly chosen object in the sample. Therefore coordinates drawn from clusters with more objects in the sample are more likely to be used for exploration.

The second heuristic to favor promising centers consists in counting for each dimension of each center the number of adjustments that have led to a reduction of the $S A E$. The algorithm keeps track of weights reflecting these counts. The weight of a center (sum of the weights of its dimensions) is then used as an evidence to encourage further the improvement of its location. In the optimization process the weights should not only increase, since when a promising center has already been optimized sufficiently it is then likely to lead to minor gains, and then should receive less attention. The weights of the dimensions in the clusters are thus decreased during the exploration, using a random selection with a probability proportional to the weights. A weight of zero for a dimension in a cluster is an evidence that it is not important to keep or adjust it, and thus 
that it should not be retained to form the subspace associated to this cluster. A non-zero weight is then interpreted as denoting a dimension being potentially meaningful for that cluster. To permit to encode a model of size $S D_{\max }$, but no more, the maximum sum of all weights is set to $S D_{\max }$.

In SubCMedians, a candidate model $\mathcal{M}$ is defined as a pair of matrices $\langle\mathcal{L}, \mathcal{W}\rangle . \quad \mathcal{L}$ and $\mathcal{W}$ describe respectively to the locations and to the weights of the centers along each dimension, both matrices having $S D_{\max }$ rows and $D$ columns. An element $\mathcal{L}_{i, d}$ represents the coordinate of a center $m_{i}$ along dimension $d$, and an element $\mathcal{W}_{i, d}$ denotes the weight of dimension $d$ for $m_{i}$. The subspace associated to a center $m_{i}$ is $\mathcal{D}_{i}=\left\{d \in\{1, \ldots, D\} \mid \mathcal{W}_{i, d}>0\right\}$. Valid centers are those for which at least one dimension is defined. Their set of indices is simply $\Gamma=\left\{c \in\left\{1, \ldots, S D_{\max }\right\} \mid \sum_{d=1}^{D} \mathcal{W}_{c, d}>0\right\}$. The total model weight, noted $\omega$, is $\sum_{i, j} \mathcal{W}_{i, j}$ and the model size is simply $\sum_{c \in \Gamma}\left|\mathcal{D}_{c}\right|$.

Example Let us consider two matrices $\mathcal{L}$ and $\mathcal{W}$ defined for $S D_{\max }=6$ and $D=2$ :

$$
\mathcal{L}=\left[\begin{array}{cc}
1 & 2 \\
0.5 & 0 \\
0 & -0.8 \\
0 & 0 \\
0 & 0 \\
-0.3 & 0.2 \\
0 & 0
\end{array}\right] \begin{aligned}
& 1 \\
& 5 \\
& 6
\end{aligned} \quad \mathcal{W}=\left[\begin{array}{ll}
1 & 2 \\
2 & 0 \\
0 & 1 \\
0 & 0 \\
0 & 0 \\
2 & 1 \\
0 & 0
\end{array}\right] \begin{aligned}
& 1 \\
& 2 \\
& 3 \\
& 5 \\
& 5
\end{aligned}
$$

Rows 3, 4 and 6 describe no valid center (all weights equal to zero in $\mathcal{W}$ ), while rows 1,2 and 5 represent the centers $m_{1}, m_{2}$ and $m_{5}$ and their subspaces: $\mathcal{D}_{1}=$ $\{1\}, \mathcal{D}_{2}=\{2\}$ and $\mathcal{D}_{5}=\{1,2\}$. The respective coordinates in their associated subspaces are given by $\mathcal{L}: m_{1}=(0.5), m_{2}=(-0.8)$ and $m_{5}=(-0.3,0.2)$.

In the algorithm given in Figure 1, the model $\mathcal{M}=\langle\mathcal{L}, \mathcal{W}\rangle$ is initialized line 3 by filling all rows of the two matrices with zeros, to represent an initial empty model. The heuristic based neighborhood exploration is performed by the successive calls to function ONE-NeIGHBOR() detailed in the next section.

\subsection{One neighbor computation function}

The function One-Neighbor () is given in Figure 2. First, it computes $\omega$ the total current model weight. Then operations carried out from lines 4 to 10 decrease the model weight when the total weight reaches the maximum model size (i.e., $\left.S D_{\max }\right)$. This is performed by picking at random a pair of indices $\langle i, j\rangle$ with a probability proportional to $\mathcal{W}_{i, j}$ (line 5 ). Then the corresponding weight $\mathcal{W}_{i, j}$ is decreased (line 6). For the sake of clarity the associated coordinate $\mathcal{L}_{i, j}$ is set to zero if $\mathcal{W}_{i, j}=0$ (line 8), even though this is not useful for the rest of the process. Next, the algorithm draws uniformly an object $s$ in the sample and a dimension $d$ in the space $\mathcal{D}$ (lines 11 and 12). Line 13 computes the set $\Gamma$ of the indices of the valid centers. Then the algorithm chooses a random center index $c$ that corresponds either to an unused center with probability $\frac{1}{\omega}$ (lines 


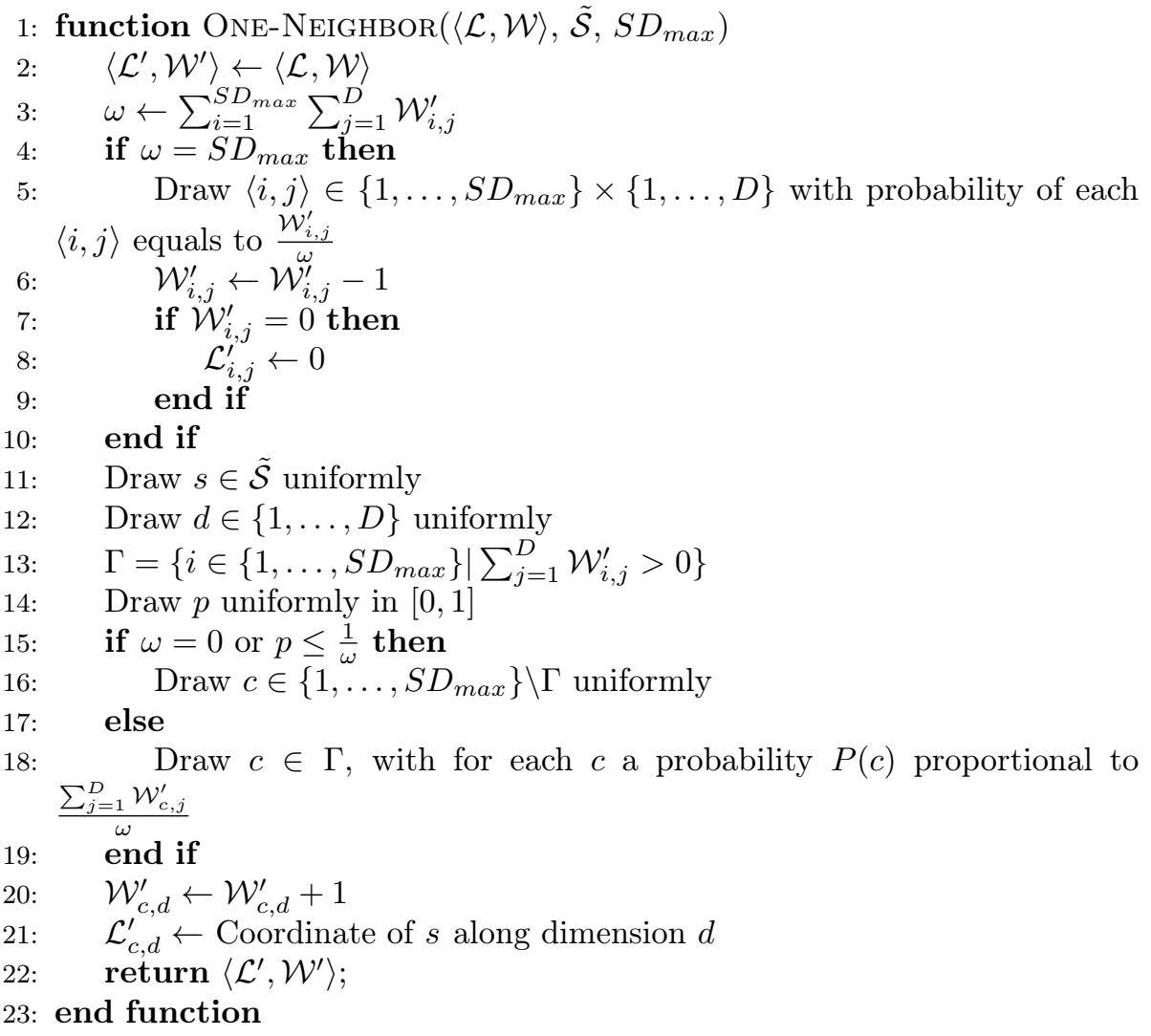

Figure 2: Generation of one neighbor.

15 and 16), or one of the current valid centers (line 18). In the latter case, the valid center is picked with a probability proportional to its total weight. Finally the weight $\mathcal{W}_{c, d}$ is increased and the location encoded in $\mathcal{L}_{c, d}$ is replaced by the coordinate of $s$ along dimension $d$.

\subsection{Complexity}

Consider one iteration of SubCMedians using sample $\tilde{\mathcal{S}}$ and let NbCenters denote the number of centers currently used (the valid centers). It can be easily derived (see Appendix A.2) that the complexity of an iteration in SubCMedians is then $\mathcal{O}(|\tilde{\mathcal{S}}| \times N b C e n t e r s \times D)$ and its memory requirement is simply $\mathcal{O}(D \times$ $\left.\left(|\tilde{\mathcal{S}}|+S D_{\max }\right)\right)$. 


\section{Experimental Setup}

\subsection{Experimental protocol}

The SubCMedians algorithm extends the subspace clustering tool family towards medians based clustering. Even if cluster center locations based on medians can be interesting on their own (depending on the targeted task), an open question is to what extent the clusters obtained make sense with respect to those given by other paradigms? We used the evaluation framework of reference designed for subspace clustering described in [13] that reports the results obtained by well-established approaches for different evaluation measures, on both real and synthetic datasets. The method presented in [13] relies on a broad exploration of the parameter space for each algorithm in order to find the best parameter setting on each data set and then compare the best results obtained by the different methods (see Appendix A.4). However such parameter tuning is most of the time a difficult task and these high quality subspace clustering models are likely to be difficult to obtain. For SubCMedians, for each dataset, a single parameter setting was used based on a default setting presented in Section 6.3. Since SubCMedians is nondeterministic (as many subspace clustering approaches), it can achieve different results on different runs. Consequently the algorithm was executed 10 times independently using the same parameter setting. The results obtained after each run were assessed with the same criteria as in [13]: the coverage, the number of clusters found, the average cluster dimensionality and different quality measures $\left(F_{1}\right.$, accuracy, $C E, R N I A$ and entropy).

All experiments were run on an Intel 2.67GHz CPU running Linux Debian 8.3, using a single core and less than $150 \mathrm{MB}$ of RAM. The SubCMedians algorithm has been implemented in $\mathrm{C}++$ (as a Python library) and is available upon request.

\subsection{Datasets}

The performances of SubCMedians are reported on real world data using the seven benchmark datasets selected in [13] for their representativity: breast, diabetes, liver, glass, shape, pendigits and vowel (most of them coming from the UCI archive [3]). These datasets have different dimensionalities and contain different numbers of objects. These objects are already structured in classes, and the class membership is used by quality measures to assess the cluster $p u$ rity. However, the number of classes does not necessarily reflect the number of subspace clusters, and the objects of a class can form different groups in space and/or strongly overlap with the objects of other classes.

SubCMedians was also executed on the 16 synthetic benchmark datasets provided by [13]. These datasets are particularly useful to study the algorithm performances, as the true clusters and their subspaces are known. Each dataset contains 10 hidden subspace clusters laying in subspaces made by $50 \%, 60 \%$ and $80 \%$ of the total dimensions of the dataset. Seven synthetic datasets allow 
to study scalability with respect to the dataset dimensionality $(5,10,15,20$, 25,50 and 75 dimensions). These datasets contain about 1500 objects each and are extended with about $10 \%$ of noise objects. Five other synthetic datasets permit to analyse the scalability with respect to the dataset size $(1500,2500$, 3500,4500 and 5500 objects), over 20 dimensions, also with about $10 \%$ of noise objects. Finally four datasets allow to study the capacity to cope with noise, containing $10 \%, 30 \%, 50 \%$ and up to $70 \%$ of noise objects.

All datasets are made available by the authors of [13] at http://dme.rwthaachen.de/openSubspace/evaluation. We applied a z-score standardization to all real and synthetic datasets in a preprocessing step.

\subsection{Parameter values}

The SubCMedians algorithm has three parameters: $S D_{\max }, N b I t e r$, and $N$. The most important is $S D_{\max }$, the maximum model size, constraining the level of detail of the subspace clustering performed. The number of iterations NbIter is also important but it should be noticed that its setting could be avoided since the user could monitor the value of the $S A E$ during the clustering and stop the process when this value does no longer improve. The third one, the sample size $N$ is a way to reduce the computing resources needed, and of course if possible it makes sense to use the full dataset instead of a sample.

In this section, we provide guidelines for easy default parameter setting. In this context, the user only needs to provide an expected or suggested number of clusters NbExpClust. Let $D$ denote the dimensionality of each particular dataset. The default setting is $S D_{\max }=N b$ ExpClust $\times D$, NbIter $=$ $10 \times S D_{\max } \times N b E x p C l u s t$ and $N=25 \times N b E x p C l u s t$ (see Appendix A.3 for justification and technical details). For the synthetic datasets the number of expected clusters is about 10, thus the maximum model size was set to $S D_{\max }=D \times 10$, the sample size was set to $N=25 \times 10$ and the number of iterations was set to NbIter $=10 \times 10 \times S D_{\max }$. A weaker setting, not based on the true number of clusters, but based on 20 expected clusters, was also used, and as reported in Section 7 still permitted to exhibit structures of about 10 clusters. For the real world datasets, the only information available about the structure of the datasets is the number of classes. However, as already mentioned, the number of classes may not correspond to the number of clusters. Indeed, according to the results provided by [13], in these datasets the state-of-the-art algorithms exhibit most of the time structures composed of more clusters than the number of classes. The setting of SubCMedians parameters is thus based on an expected number of clusters equal to three times the number of classes and we let the algorithm regulate itself the number of output clusters. Hence the maximum model size was set to $S D_{\max }=3 \times N$ NClasses $\times D$, the sample size was set to $N=25 \times 3 \times N b C l a s s e s$ and the number of iterations was set to $N b I t e r=10 \times 3 \times N b C l a s s e s \times S D_{\max }$. 


\subsection{Evaluation measures}

In order to compare our algorithm to the others, we used the same standard evaluation measures for clusters and subspace clusters as in [13]: entropy (the normalized entropy), accuracy, $F 1, R N I A$ and $C E$. We performed also the same simple transformation on entropy and $R N I A$, by computing $\overline{R N I A}=1-$ $R N I A$ and $\overline{\text { entropy }}=1$-entropy to have all evaluation measures ranging from 0 (low quality) to 1 (high quality). The three first measures (entropy, accuracy and $F 1$ ) reflect how well objects that should have been grouped together were effectively grouped. The two last measures $(\overline{R N I A}$ and $C E)$ take into account the way the objects are grouped and also the relevance of the subspaces found by the algorithm. For these measures, when the true dimensions of the subspace clusters were not known (for real datasets), then as in [13] all dimensions were considered as relevant, but then the interpretation of these two measures should remain cautious since the true sets of dimensions are likely to be smaller. Of course this does not apply to the synthetic datasets, since for them the reference clusters and their dimensions are known. We refer the reader to [13] for the detailed recall of the evaluation measures.

\section{$7 \quad$ Experimental Results}

Because of the large number of results (23 datasets and 11 algorithms) used in the comparison, aggregated figures reflecting the real detailed results are given. However, there is no ever winning paradigm or clustering method, each having its own advantages or interest.

\subsection{Real dataset}

This section presents the results obtained on the 7 representative real datasets selected in [13]. In order to assess if SubCMedians produced decent results with respect to well-established methods, we ranked the different methods with respect to their quality measures, their coverage (fraction of objects of the dataset that were grouped into clusters) and the number of clusters they produced (see Appendix A.5 for a detailed presentation of this procedure).

The average rank of each algorithm regarding the cluster quality measures, the coverage and the number of clusters found are given in Figure 3 (highest ranks meaning best results). In addition, two tables representative of the results at a more detailed level are presented in Figure 4. These tables report on two of the real world datasets the values for the coverage, the number of clusters found, their average dimensionality and the runtimes. Runtimes are given only as complementary information since for SubCMedians they were obtained on a computer $(2.67 \mathrm{GHz} \mathrm{CPU})$ different from the one used by [13] (2.3GHz CPU). At the bottom of these tables, we grouped the results obtained by methods belonging to the same family as SubCMedians (clustering-oriented techniques), results for the other algorithms are provided for the sake of completeness. 

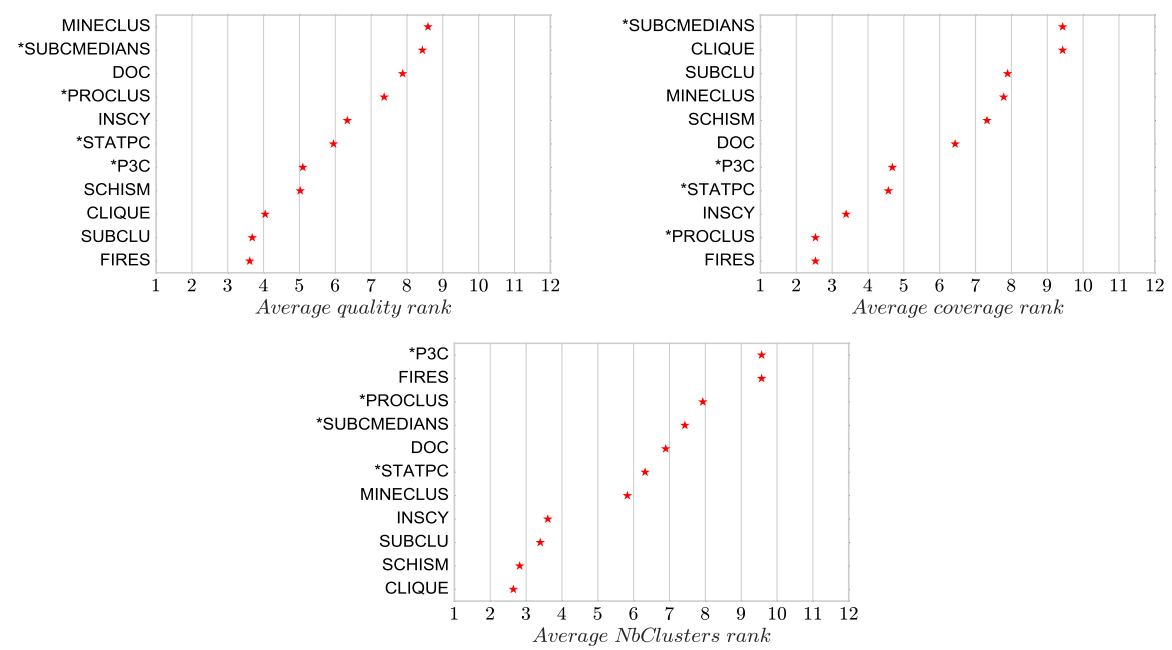

Figure 3: Average rankings over all datasets regarding the quality, the coverage and the number of clusters.

Figures 3 and 4 show evidence that the robustness of the medians still operates in this subspace clustering task since competitive cluster qualities can be obtained without discarding any object (100\% coverage). In all experiments SubCMedians was run using the default parameter setting. For the number of clusters found, SubCMedians is above the average, and it seems that it does not tend to split the dataset into too many clusters. However, the true numbers of clusters are not known here, in contrast to the synthetic datasets.

\subsection{Synthetic data}

In this section, we report the results obtained on the 16 synthetic datasets of [13], each one containing 10 known clusters. For each dataset, SubCMedians was executed 10 times and the model having the lowest SAE was retained (no use of external labeling). These models are depicted as red circles in Figure 7 (blue triangles correspond to models obtained with an unweighted version of SubCMedians discussed here after). The green shapes represent the areas where are located the best models found by the 10 other algorithms over their parameter spaces (optimized using external ground truth labeling). Here again, the results show evidences that a median based approach is an interesting complementary tool for subspace clustering, and in particular that SubCMedians reaches a competitive quality for the measures that take into account not only the objects grouping, but also the dimensions associated to the clusters $(\overline{R N I A}$ and $C E$ ). Runtimes as functions of the size of the sample and the dimensionality of the dataset are provided in Figure 6. In these experiments the parameters where set according to the guidelines of Section 6.3 using an expected number 


\begin{tabular}{|c|c|c|c|c|c|c|c|c|}
\hline \multicolumn{9}{|c|}{ Pendigits: 16 dimensions, 10 classes, 7494 objects } \\
\hline & \multicolumn{2}{|c|}{ Coverage } & \multicolumn{2}{|c|}{ Cluster Number } & \multicolumn{2}{|c|}{ Average Dim } & \multicolumn{2}{|c|}{ Runtime (seconds) } \\
\hline & $\max$ & & $\max$ & $\min$ & $\max$ & & $\max$ & $\min$ \\
\hline CLIQUE & 1.00 & 1.00 & 1890.0 & 36.0 & 3.10 & 1.50 & 67891 & 219 \\
\hline DOC & 0.91 & 0.91 & 15.0 & 15.0 & 5.50 & 5.50 & 178358 & 178358 \\
\hline MIN & 1.00 & 1.00 & 64.0 & 64.0 & 12.10 & 12.10 & 780167 & 692651 \\
\hline & 1.00 & 0.93 & 1092.0 & 290.0 & 10.10 & 3.40 & 500000000 & 21266 \\
\hline & \multicolumn{8}{|c|}{ Runtime exceeding the maximal runtime allowed } \\
\hline FIR] & 0.94 & 0.94 & 27.0 & 27.0 & 2.50 & 2.50 & 169999 & 169999 \\
\hline INSCY & 0.91 & 0.82 & 262.0 & 106.0 & 5.30 & 4.60 & 2000000 & 1000000 \\
\hline PROCLUS & 0.90 & 0.74 & 37.0 & 17.0 & 14.00 & 8.00 & 6045 & 4250 \\
\hline P3C & 0.90 & 0.90 & 31.0 & 31.0 & 9.00 & 9.00 & 2000000 & 2000000 \\
\hline STATPC & 0.99 & 0.84 & 4109.0 & 56.0 & 16.00 & 16.00 & 50000000 & 3000000 \\
\hline SUBCMEDIANS & 1.00 & 1.00 & 41.0 & 34.0 & 12.21 & 10.51 & 556 & 523 \\
\hline
\end{tabular}

\begin{tabular}{|c|c|c|c|c|c|c|c|c|}
\hline & \multicolumn{6}{|c|}{ Diabetes: 8 dimensions, 2 classes, 768 objects } & \multirow{2}{*}{\multicolumn{2}{|c|}{ Runtime (seconds) }} \\
\hline & \multicolumn{2}{|c|}{ Coverage } & \multicolumn{2}{|c|}{ Cluster Number } & \multicolumn{2}{|c|}{ Average Dim } & & \\
\hline & $\max$ & & $\max$ & $\min$ & $\max$ & $\min$ & $\max$ & $\min$ \\
\hline CLIQUE & 1.00 & 1.00 & 349.0 & 202.0 & 4.20 & 2.40 & 11953 & 203 \\
\hline DOC & 1.00 & 0.93 & 64.0 & 17.0 & 8.00 & 5.10 & 1000000 & 51640 \\
\hline MINECLUS & 0.99 & 0.96 & 39.0 & 3.0 & 6.00 & 5.20 & 3578 & 62 \\
\hline SCHISM & 1.00 & 0.79 & 270.0 & 21.0 & 4.20 & 3.90 & 35468 & 250 \\
\hline SUBCLU & 1.00 & 1.00 & 1601.0 & 325.0 & 4.70 & 4.00 & 190122 & 58718 \\
\hline FIRES & 0.81 & 0.03 & 17.0 & 1.0 & 2.50 & 1.00 & 4234 & 360 \\
\hline INSCY & 0.83 & 0.73 & 132.0 & 3.0 & 6.70 & 5.70 & 112093 & 33531 \\
\hline PROCLUS & 0.92 & 0.78 & $\overline{9.0}$ & 3.0 & $\overline{8.00}$ & 6.00 & 360 & 109 \\
\hline P3C & 0.97 & 0.88 & 2.0 & 1.0 & 7.00 & 2.00 & 656 & 141 \\
\hline STATPC & 0.97 & 0.75 & 363.0 & 27.0 & 8.00 & 8.00 & 27749 & 4657 \\
\hline SUBCMEDIANS & 1.00 & 1.00 & 16.0 & 13.0 & 3.69 & 2.87 & 3 & 3 \\
\hline
\end{tabular}

Figure 4: Details of the cluster structures and of the runtimes on Pendigits and Diabetes.

of clusters of 10, but no constraint on the sizes of their subspaces. Interestingly, a weaker setting based on an expected number of clusters of 20 still permitted to SubCMedians to exhibit most of the time a good quality clustering of about 10 clusters (not shown, very similar to Figure 7).

Figure 5 illustrates the clustering quality in terms of $\overline{R N I A}$ for increasing percentages of noise points in the dataset (respectively 10,30, 50 and $70 \%$ of the data points were noise points). Even if the quality of the subspace clusters obtained tends to decrease when the amount of noise in the dataset increases, the degradation is not severe. Here again, this seems to indicates that the robustness to noise of the medians still operates in a subspace clustering task.

In order to assess the impact of the weighted neighborhood exploration of the model space used by SubCMedians, the algorithm was compared to an unweighted version. In this version, the candidate center undergoing the modification is uniformly drawn by modifying line 18 in Figure 2, and in the same figure line 20 is replaced by $\mathcal{W}_{c, d}^{\prime} \leftarrow 1$ to have weight matrices that contain simply a 1 for a dimension that is used and a 0 otherwise.

The results of the more naïve unweighted version of SubCMedians are represented in Figure 7 by blue triangles (same procedure used, retaining also the lowest SAE over 10 runs). The unweighted-SubCMedians outputted slightly more clusters than expected, and has another more important drawback, that is the lower quality obtained with respect to the dimensions found $\overline{(R N I A}$ 


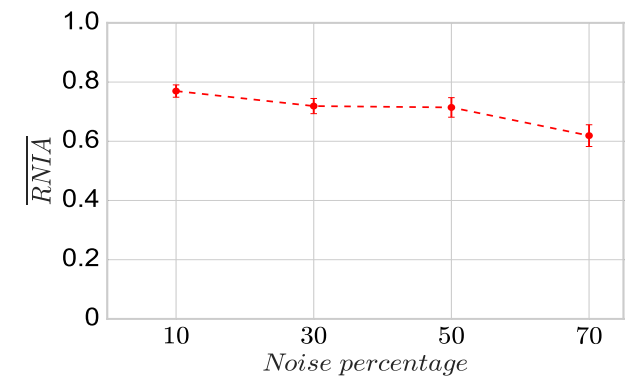

Figure 5: Mean $\overline{R N I A}$ vs percentage of noise points in the dataset.

and $C E$ graphics). As targeted by the design of the neighborhood exploration in Section 5, the weighted strategy of SubCMedians could focus on more promising clusters while unweighted-SubCMedians produced more (useless) candidate centers (Figure 8) and failed to develop sufficiently the center dimensionalities when the hidden cluster dimensionalities increased (Figure 9). Moreover, SubCMedians turned out to be slightly faster (see Figure 6), and unweighted-SubCMedians suffered from a small overhead likely to be caused by its handling of more candidate centers in the models.

\section{Conclusion}

In this paper we presented SubCMedians, a median-based subspace clustering method, and assessed it on real and synthetic benchmark datasets, using the evaluation framework of [13]. These results showed that a median-based subspace clustering approach can exhibit satisfactory results when compared to well-established subspace clustering paradigms, and is thus a good candidate as a complementary tool, in particular for users interested by the properties of medians themselves (facility location, robustness to noise and outliers). We also proposed some guidelines for easy default parameter setting. These guidelines were effective when dealing with all the datasets of the benchmark. Since the median-based subspace clusters seem to be obtained in rather short runtimes, promising future work includes the use of these clusters as initial guesses to guide other techniques that could be more time consuming.

\section{A Appendix}

\section{A.1 Expected gain in SAE to guide local exploration}

Consider a cluster $C$ and a dimension $d$. Let us suppose that the coordinates $X_{d}$ of the objects in $C$ along $d$ follow a Gaussian distribution $\mathcal{N}\left(\mu_{d}, \sigma_{d}\right)$. Let us consider that a badly placed center $m$ is simply an object in $C$ taken randomly. Along dimension $d$, the difference between the location of an object $X$ chosen 


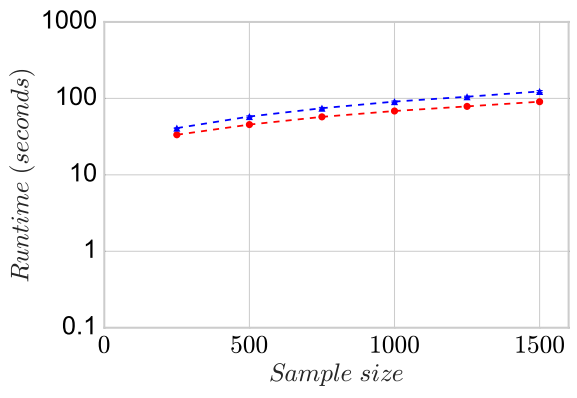

(a) Runtime vs. dataset sample size.

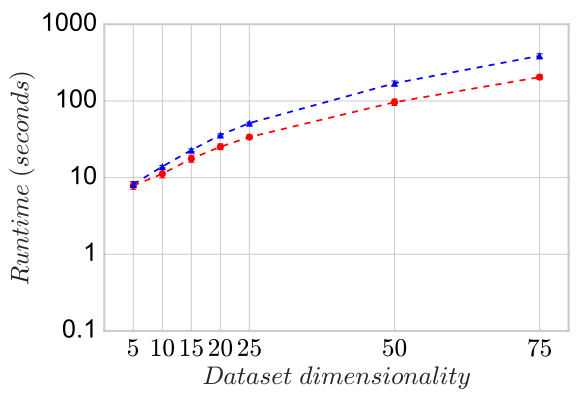

(b) Runtime vs. dataset dimensionality

Figure 6: Runtimes (avg. 10 runs) for SubCMedians vs sample sizes $(|\tilde{\mathcal{S}}|)$ for the synthetic dataset of size 5500 (6a), and vs the synthetic dataset dimensionalities (6b). Runtimes of the unweighted version of SubCMedians as blue triangles. 

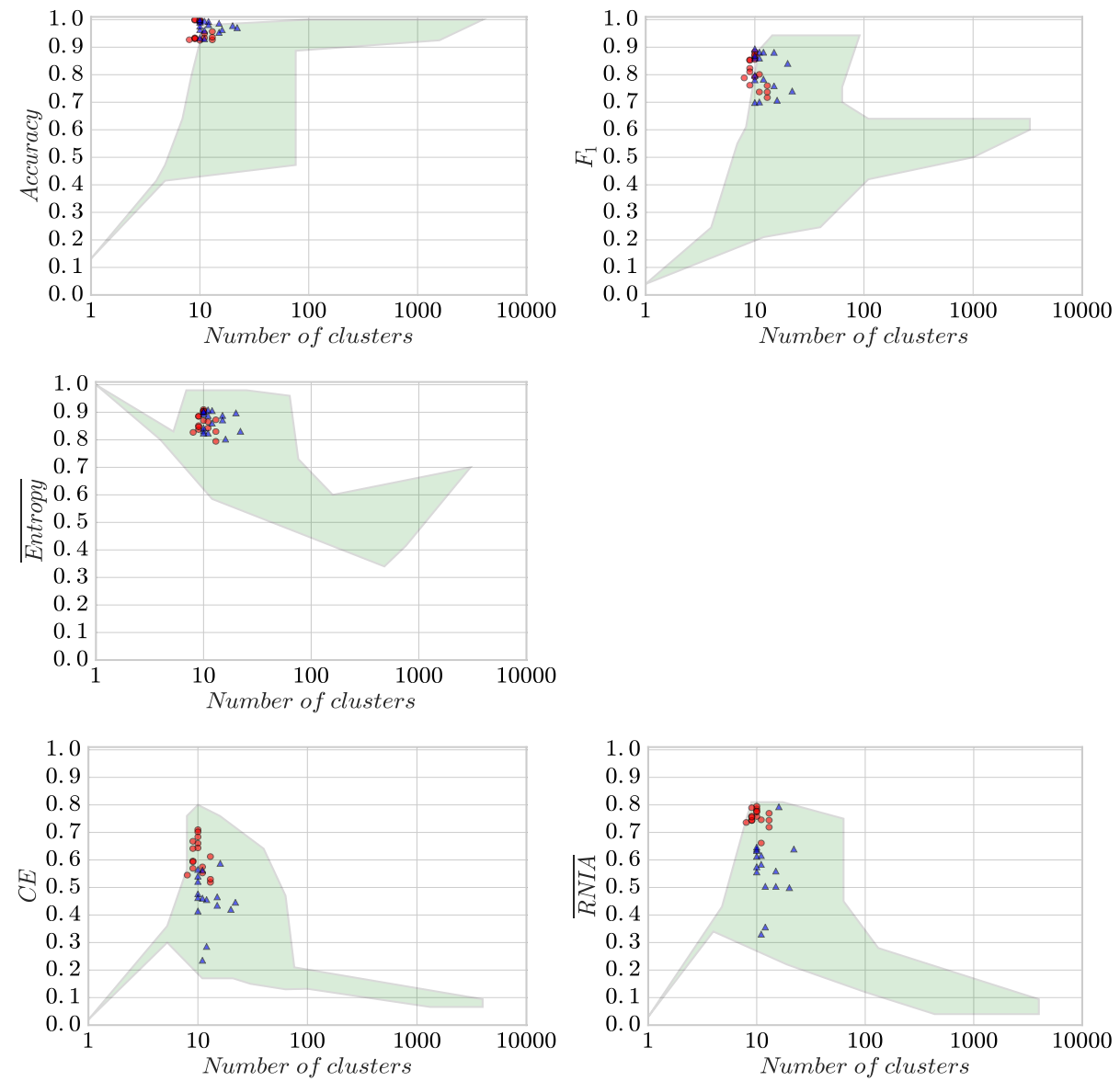

Figure 7: Quality measures and number of clusters obtained on synthetic datasets by SubCMedians (red circles) and the other algorithms (green areas). Results of the unweighted version of SubCMedians as blue triangles. 
randomly in $C$ and the location of $m$, is $\left(X_{d}-m_{d}\right)$ and follows the distribution $\mathcal{N}\left(\mu_{d}^{\prime}, \sigma_{d}^{\prime}\right)$ with $\mu_{d}^{\prime}=0$ and $\sigma_{d}^{\prime}=\sigma_{d} \sqrt{2}$ since $X_{d}$ and $m_{d}$ follows $\mathcal{N}\left(\mu_{d}, \sigma_{d}\right)$.

Let us consider that a well placed center $m^{*}$ is the median of the $n$ objects of $C$ contained in the current sample $\tilde{\mathcal{S}}$. Using the distribution followed by such a median estimator and obtained in Section 4.2 we have $m^{*} \sim \mathcal{N}\left(\mu_{d}, \sigma_{d} \sqrt{\frac{\pi}{2 n}}\right)$. Thus the difference between the locations of object $X$ and $m^{*}$ along $d,\left(X_{d}-m_{d}^{*}\right)$, follows the distribution $\mathcal{N}\left(\mu_{d}^{*}, \sigma_{d}^{*}\right)$ with $\mu_{d}^{*}=0$ and $\sigma_{d}^{*}=\sigma_{d} \sqrt{\frac{\pi}{2 n}+1}$.

For $m$ the contribution of $X$ to the AE value (and thus to $S A E$ ) is $\mid X_{d}-$ $m_{d} \mid$. As $\left(X_{d}-m_{d}\right) \sim \mathcal{N}\left(\mu_{d}^{\prime}, \sigma_{d}^{\prime}\right)$, then $\left|X_{d}-m_{d}\right|$ follows the corresponding folded normal distribution and the expected value of $\left|X_{d}-m_{d}\right|$ is given by $\sigma_{d}^{\prime} \sqrt{2 / \pi} \exp \left(-\mu_{d}^{\prime 2} / 2 \sigma_{d}^{\prime 2}\right)+\mu_{d}^{\prime}\left[1-2 \Phi\left(-\mu_{d}^{\prime} / \sigma_{d}^{\prime}\right)\right]$, where $\Phi$ denotes the normal cumulative distribution. Since $\mu_{d}^{\prime}=0$ and $\sigma_{d}^{\prime}=\sigma_{d} \sqrt{2}$, we have $\mathbb{E}\left(\left|X_{d}-m_{d}\right|\right)=$ $2 \sigma_{d} / \sqrt{\pi}$. In a similar way we can derive the expected value of the contribution of $X$ to $A E$ for the optimized center $m^{*}, \mathbb{E}\left(\left|X_{d}-m_{d}^{*}\right|\right)=\sigma_{d} \sqrt{\frac{1}{n}+\frac{2}{\pi}}$.

If we consider $\gamma=\mathbb{E}\left(\left|X_{d}-m_{d}^{*}\right|\right)-\mathbb{E}\left(\left|X_{d}-m_{d}\right|\right)$ as reflecting the gain due in the optimized case, then $\gamma=\sigma_{d}\left(2 / \sqrt{\pi}-\sqrt{\frac{1}{n}+\frac{2}{\pi}}\right)$. This means that larger gains are likely to be obtained for clusters having a high $\sigma_{d}$ and having many of their elements belonging to the sample $\tilde{\mathcal{S}}$.

\section{A.2 Complexity}

Consider one iteration of SubCMedians using sample $\tilde{\mathcal{S}}$ in a $D$ dimensional space. Let $N b C e n t e r s$ denote the number of centers currently used (the valid centers). Operations in non-constant time are the calls to $A E, S A E$ and ONENeighbor(). $A E$ computes the distance of one object to each center and is in $\mathcal{O}(N b C e n t e r s \times D)$. $S A E$ does the same for each object in the sample and then has a complexity $\mathcal{O}(|\tilde{\mathcal{S}}| \times N b C e n t e r s \times D)$. The computation cost of ONENeighbor() lies in part in the computation of weights: the total weight of the model $\omega$ (line 3 ) and the weights in the probabilities used line 18. These values can be maintained incrementally when $\mathcal{W}$ is modified resulting in a constant cost for $\omega$ and in a cost proportional to NbCenters for the probabilities line 18. The remaining cost is due to the non-uniform random selections (lines 5 and 18), having a cost proportional to the number of possible outcomes. Line 5 there are at most $N b C$ enter $\times$ $D$ possible outcomes with a non-zero probability (other pairs $\langle i, j\rangle$ having a weight of zero). And for line 18 there are NbCenters possible outcomes. The cost of one call to One-Neighbor() is thus in $\mathcal{O}(N b C e n t e r s \times D)$. The complexity of an iteration in SubCMedians is then $\mathcal{O}(|\hat{\mathcal{S}}| \times N$ bCenters $\times D)$.

The memory requirement of one iteration of SubCMedians corresponds to the storage of the sample $\tilde{\mathcal{S}}$ and the two matrices $\mathcal{L}$ and $\mathcal{W}$ of size $S D_{\max } \times D$ and thus is simply in $\mathcal{O}\left(|\tilde{\mathcal{S}}| \times D+S D_{\max } \times D\right)$. 


\section{A.3 Parameter Setting}

The easy default parameter setting (for preliminary exploration of the data) requires to provide only one value: the expected number of clusters NbExpClust. Notice that this is not a crisp constraint, but only a suggested number of clusters that the algorithm should adjust to build a structure. Notice also that a hard part of the subspace clustering task is to determine the subspaces and that this aspect is not directly constrained by NbExpClust.

Maximum model size $S D_{\max }$ The intuition to set $S D_{\max }$ is very simple, the value of $S D_{\max }$ should allow to build a model containing NbExpClust even if all dimensions are useful, leading to a default $S D_{\max }$ value equal to NbExpClust $\times D$. Of course, as for any subspace clustering algorithm, obtaining very satisfactory results are likely to require from the user finer parameter tuning.

From $N b E x p C l u s t$ and $S D_{\max }$ we now derive minimum recommended values for the number of iterations and for the sample size. These settings are the ones used in the experiments reported Section 7, but of course the statistical thresholds used in the setting method could be enforced in order to have more conservative recommended parameter values.

Number of iterations NbIter Let $C$ be any expected cluster and $d$ be any of its dimensions. Let $k$ be the number of attempts to set the coordinate of the center of $C$ along $d$ to a reasonable approximation of the median value, during NbIter iterations in SubCMedians. The default value proposed for NbIter is the minimum number of iterations such that the expected value of $k$ is at least 1.

Let us suppose that all clusters have the same size and that the number of clusters is equal to NbExpClust. Consider an iteration of SubCMedians. In OnE-NeIGHBOR() (Figure 2) the probability to pick an object $s$ belonging to $C$ (line 11) is $1 / N b \operatorname{ExpClust}$ and the probability to choose dimension $d$ (line 12) is $1 / D$. Let $x$ be the coordinate of $s$ along dimension $d$, and let us accept as a reasonable approximation a value $x$ at a distance of less than $1 / 8$ of the standard deviation away from the median. Then, supposing a Gaussian distribution, this implies that the median is equal to the mean, and that the probability of $x$ as being a reasonable approximation is about 0.1 (according to the standard normal cumulative distribution). At line 15, the probability to jump to line 18 to modify an existing cluster is $1-1 / \omega$. If we suppose that the current model contains already $N b E x p C l u s t$ clusters, including $C$ (possibly with the presence of a single dimension of $C$ ), and that all clusters have similar weights in matrix $\mathcal{W}$, then the probability of $C$ to be chosen in line 18 is about $1 /$ NbExpClust. So the probability $p$ of the center corresponding to $C$ in the current model, to have its coordinate along dimension $d$ to be set to a reasonable approximation $x$ in line 21 , is $p=\frac{1}{\text { NbExpClust }} \times \frac{1}{D} \times 0.1 \times\left(1-\frac{1}{\omega}\right) \times \frac{1}{\text { NbExpClust }}$. As $\omega$ increases at each iteration (up to $S D_{\max }$ ) we quickly have $\left(1-\frac{1}{\omega}\right) \simeq 1$, and since we set $S D_{\max }=N b E x p$ Clust $\times D$, we have $p \simeq 0.1 \times \frac{1}{S D_{\max } \times N b E x p C l u s t}$. 
The expected value of $k$ is $\mathbb{E}(k)=$ NbIter $\times p$, thus to have $\mathbb{E}(k) \geq 1$, the default minimum NbIter value is set to $10 \times S D_{\max } \times N b$ ExpClust.

Dataset sample size $N$ Consider a cluster $C$ and a dimension $d$, supposing that the coordinates in $C$ along $d$ follow $\mathcal{N}\left(\mu_{d}, \sigma_{d}\right)$. As stated in Section 4.2, $\hat{\theta}_{d}$ the median estimator of these coordinates, using a sample of size $n$, follows $\mathcal{N}\left(\mu_{d}, \sigma_{d} \sqrt{\frac{\pi}{2 n}}\right)$. If we accept a standard error of $1 / 4$ of the original $\sigma_{d}$ then the minimum sample size satisfies $\sigma_{\hat{\theta}_{d}}=\frac{\sigma_{d}}{4}=\sigma_{d} \sqrt{\frac{\pi}{2 n}}$ and we have $n=8 \pi \simeq$ 25 . Such sample allows to estimate the median location of a cluster in the $D$ dimensions, but if there are NbExpClust clusters, more objects are needed. Let us suppose that all clusters have the same size, and thus are likely to be represented by similar numbers of objects in the sample, then the recommended minimum value for $N$ is $25 \times N b$ ExpClust.

\section{A.4 Evaluation framework}

The evaluation framework of [13] relies on a systematic approach to compare the results of representative algorithms that address the major subspace clustering paradigms. The comparison detailed in [13] was made using different evaluation measures on both real and synthetic datasets, using the following method. Given that each algorithm requires several parameters (from 2 to 9), for each dataset, the algorithms were executed with 100 different parameter settings to explore the parameter space. Then, using an external labeling of the objects, only the outputs that were among the best with respect to the external labeling (taken as a ground truth) were retained. So, the results reported in [13] are in some sense the best possible subspace clusterings that could be achieved if we were able to find the most appropriate parameter values. More precisely, for each real world dataset only two outputs were retained: 1) the one computed for the parameter setting that maximizes the $F_{1}$ measure, and 2) the one obtained when maximizing the accuracy. These two outputs led to two values for each measure, the smallest of the two being called bestMin and the other bestMax. For each synthetic dataset, all the settings maximizing at least one quality measures among $F_{1}$, accuracy, CE, RNIA and entropy were retained. bestMin and bestMax are reported as min and max in Figure 4.

Since generally no external labeling is available when we search for clusters, parameter tuning is most of the time a difficult task and these high quality subspace clustering models are likely to be hard to obtain. Instead, for SubCMedians, the parameters were not optimized using any external criteria and were set to the default values suggested in Section A.3. For each real world dataset, the highest and lowest values of each evaluation measure over 10 runs were computed, so as to compare them to the bestMax and bestMin values retained by the evaluation method of [13]. For each synthetic dataset, we simply took the clustering having the lowest $S A E$ measure (internal criterion of quality). 


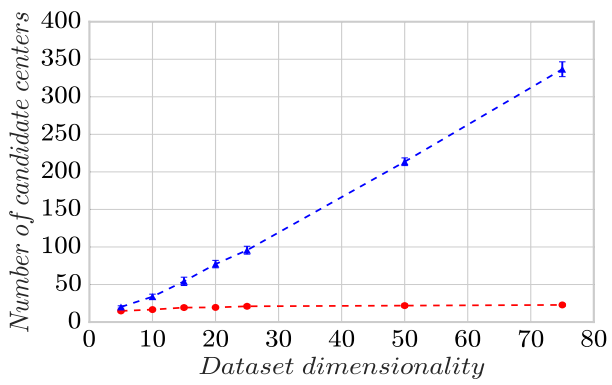

Figure 8: Number of candidate centers (avg. 10 runs) for SubCMedians (red circles) and unweighted-SubCMedians (blue triangles) vs dataset dimensionality.

\section{A.5 Ranking method over real world datasets}

The algorithms were ranked over the seven real world datasets using the following procedure.

Cluster quality measures For each measure, on each data set, two rankings of the 11 algorithms were computed: one for the bestMax score and one for the bestMin score (see Appendix A.4), with ranks ranging from 1 to 11, rank 11 being associated to the algorithm obtaining the highest quality. The overall ranking of an algorithm was then simply the average of its rank values.

Coverage The same procedure was used to compare the coverages obtained. The coverage denotes the fraction of objects of the dataset that are associated to clusters. This measure is less than 1 if some objects are not associated to one of the output clusters, or if they are identified as outliers or as reflecting noise. When the coverage decreases, discarding some objects can result in a direct improvement of several quality measures (because of clusters being more homogeneous). Of course putting apart too many objects is likely to lead to less representative models and is most of the time not desirable. For the coverage a rank value of 11 was associated to the highest coverage.

Number of clusters The rankings were computed using the absolute value of the difference between the number of clusters found by the algorithm and the number of classes in the dataset. The rank value 11 is given to the smallest absolute value. However, as previously mentioned, the number of classes does not necessarily reflect the number of subspace clusters in the datasets.

The global trade-off is then to reach high quality measures while preserving a high coverage (except for data containing many outliers or important noise), and without splitting the data into too many clusters. 


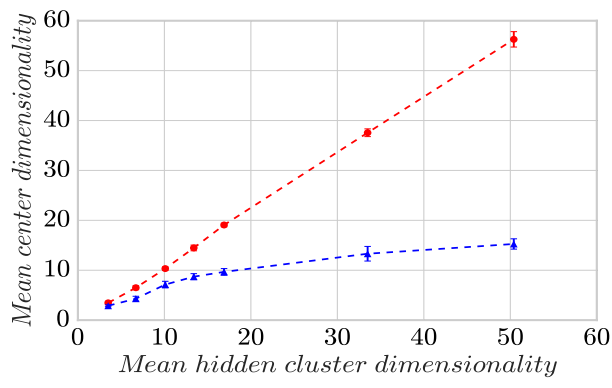

Figure 9: Subspace mean size of the centers (avg. 10 runs) for SubCMedians (red circles) and unweighted-SubCMedians (blue triangles) vs subspace mean size of the hidden clusters.

\section{B Acknowledgements}

This research has been supported by EU-FET grant EvoEvo (ICT-610427). Other support: Christophe Rigotti is a member of LabEx IMU (ANR-10-LABX-0088).

\section{References}

[1] C. C. Aggarwal, A. Hinneburg, and D. A. Keim. On the surprising behavior of distance metrics in high dimensional space. In Proc. of ICDT, pages 420-434, 2001.

[2] C. C. Aggarwal, J. L. Wolf, P. S. Yu, C. Procopiuc, and J. S. Park. Fast algorithms for projected clustering. In Proc. of ACM SIGMOD, pages 61-72, 1999.

[3] K. Bache and M. Lichman. UCI machine learning repository, 2013.

[4] S. Har-Peled and A. Kushal. Smaller coresets for K-median and K-means clustering. Discrete $\&$ Computational Geometry, 37(1):3-19, 2006.

[5] K. Jain and V. V. Vazirani. Approximation algorithms for metric facility location and K-median problems using the primal-dual schema and lagrangian relaxation. Journ. of ACM, 48(2):274-296, 2001.

[6] M. Jamshidi. Median location problem. In R. Zanjirani Farahani and M. Hekmatfar, editors, Facility Location: Concepts, Models, Algorithms and Case Studies, pages 177-191. Physica-Verlag, 2009.

[7] H.-P. Kriegel, P. Kröger, and A. Zimek. Clustering high-dimensional data: A survey on subspace clustering, pattern-based clustering, and correlation clustering. ACM TKDD, 3(1):1-58, 2009.

[8] H.-P. Kriegel, P. Kröger, and A. Zimek. Subspace clustering. Wiley Interdisciplinary Reviews: Data Mining and Knowledge Discovery, 2(4):351-364, 2012.

[9] K. H. Lim, L. Ferraris, M. E. Filloux, B. J. Raphael, and W. G. Fairbrother. Using positional distribution to identify splicing elements and predict pre-mrna processing defects in human genes. Proc. of the National Academy of Sciences, 108(27):11093-11098, 2011. 
[10] Y. Liu, L. C. Jiao, and F. Shang. An efficient matrix factorization based low-rank representation for subspace clustering. Pattern Recognition, 46(1):284-292, 2013.

[11] J. S. Maritz and R. G. Jarrett. A note on estimating the variance of the sample median. Journ. of the American Statistical Association, 73(361):194-196, 1978.

[12] R. Maronna, D. Martin, and V. Yohai. Robust statistics. John Wiley \& Sons, 2006.

[13] E. Müller, S. Günnemann, I. Assent, and T. Seidl. Evaluating clustering in subspace projections of high dimensional data. In Proc. of VLDB, pages 12701281, 2009.

[14] L. Parsons, E. Haque, and H. Liu. Subspace clustering for high dimensional data: A review. ACM SIGKDD Explorations Newsletter, 6(1):90-105, 2004.

[15] G. Sen, M. Krishnamoorthy, N. Rangaraj, and V. Narayanan. Exact approaches for static data segment allocation problem in an information network. Computers \& Operations Research, 62:282-295, 2015.

[16] S. J. Sheather. A finite sample estimate of the variance of the sample median. Statistics \& Probability Letters, 4(6):337-342, 1986.

[17] K. Sim, V. Gopalkrishnan, A. Zimek, and G. Cong. A survey on enhanced subspace clustering. DMKD, 26(2):332-397, 2013.

[18] Y. Wang and J. Zhu. Dp-space: Bayesian nonparametric subspace clustering with small-variance asymptotics. In Proc. of ICML, pages 1-9, 2015. 